\title{
Relationship between Intelligence Beliefs and Achievement Motivation with Self-regulated Learning in Students with Veteran Parents Injured More than 40\%
}

\section{ART ICLE INF O}

\section{Article Type}

Descriptive Study

\section{Authors}

Seadatee Shamir A.* $P h D$,

Sanee'I Hamzanlouyi R. ${ }^{1} M S c$

How to cite this article
Seadatee Shamir A, Sanee'I Ha-
mzanlouyi R. Relationship betw-
een Intelligence Beliefs and Ac-
hievement Motivation with Self-
regulated Learning in Students
with Veteran Parents Injured M-
ore than 40\%. Iranian Journal
of War \& Public Health. 2017;
$9(4): 205-210$.

*Educational \& Personality Psychology Department, Humanity \& Social Sciences Faculty, Science \& Research Branch, Islamic Azad University, Tehran, Iran

${ }^{1}$ Educational Psychology Department, Psychology \& Educational Sciences Faculty, Bojnord Branch, Islamic Azad University, Bojnord, Iran

\section{Correspondence}

Address: Islamic Azad University, Science \& Research Branch, Hesarek Boulevard, Daneshgah Square, The end of Sattary Highway, Tehran, Iran. Post Box: 775/14515

Phone: +98 (21) 46049687 -

Fax: +98 (21) 46049687

seadatee@gmail.com

\section{Article History}

Received: February 4, 2017

Accepted: July 19, 2017

ePublished: November 6, 2017

\section{A B S T R A C T}

Aims Students with veteran parents are encountered with issues that are differed from those for normal students. The use of self-regulated learning strategies affects students' performance. The present study was aimed to investigate the relationship between intelligence beliefs and achievement motivation with self-regulated learning of students with veteran parents injured more than $40 \%$.

Instruments \& Methods In this descriptive-correlational study, 230 students with veteran parents injured more than 40\% were selected from secondary high-schools of Bojnord city in academic year of 2014-15. A multistage-cluster sampling method was employed to select the students. This research was done utilizing three instruments including: self-regulated learning questionnaire, intelligence beliefs scale and Herman's achievement motivation scale. The data were analyzed by SPSS 23 software using Pearson correlation coefficient, multivariate and stepwise regression analyses.

Findings There were significant positive correlations between intelligence beliefs and achievement motivation with self-regulated learning $(\mathrm{p}<0.05)$. Based on the results, 24\% of variance in self-regulated learning was explained by intelligence beliefs. The other subvariables including intrinsic, incremental, educational, contextual factors and achievement motivation added $2 \%, 2 \%, 1 \%, 2 \%$ and $10 \%$ to the variance, respectively. Collectively, these six models explained $41 \%$ of the variance of self-regulated learning $(\mathrm{p}<0.01)$.

Conclusion Intelligence beliefs and achievement motivation predict self-regulation learning in students with veteran parents injured more than $40 \%$.

Keywords Intelligence Belief; Achievement Motivation; Self-Regulation Learning; Student with $40 \%$ Veteran Parents

\section{I T A T I O N L I N KS}

[1] Investigating the relationship between cognitive and metacognitive skills with Locus of control and achievement motivation in ... [2] Comparison of motivational beliefs, selfregulation strategies and personality factors and their predictive role on ... [3] Investigating the effect of self- regulated learning strategies training on female firstgrade high school students' math learning and anxiety in ... [4] The role of personality factors, learning approaches and thinking styles on academic ... [5] The relationship between personality characteristics, intelligence beliefs and academic achievement goals ... [6] The relationship between intelligence beliefs and goals of progress with selfefficacy in ... [7] Survey students self-monitoring and parents learning: The study of gender and ... [8] Motivation and academic achievement in medical ... [9] The relation between self regulated learning... [10] A comparison of social, emotional and educational adjustment ... [11] The effect of educational and self-regulatory methods ... [12] The relationship between perception of parenting styles and ... [13] The relationship between intelligence beliefs, achievement ... [14] The role of dimensions of perfectionism and ... [15] Standardize training persuasion questionnaire ... [16] Self-esteem and achievement motivation ... [17] The relationship between self-regulation ... [18] Motivation and student's use of learning strategies ... [19] The relationship between the big five model ... [20] Handbook of self- regulation. San Diego ... [21] Self- regulation on a concept information's ... [22] Self-regulated learning: From teaching ... [23] The "what" and "why" of goal pursuits ... [24] Applying educational ... [25] Latent ability: grades and test scores systematically ... [26] Self-regulated ... [27] Study of achievement motivation in relation to academic achievement ... [28] Further investigating thinking styles and psychosocial ... [29] Investigating self-regulation and motivation ... 


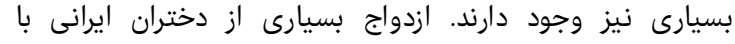

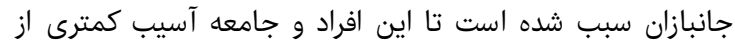
اين مجروحيتها متحمل شوند [1] إن.

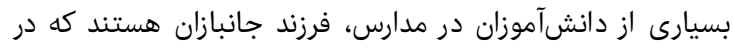

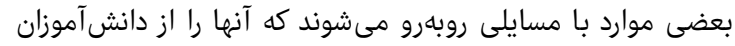

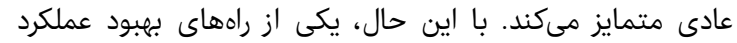

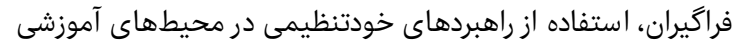

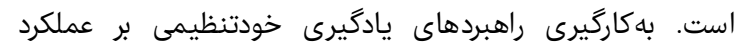

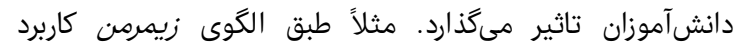

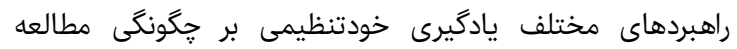

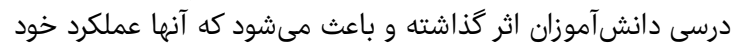

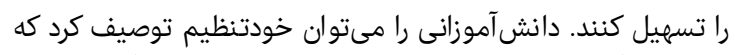

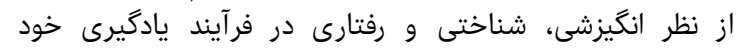

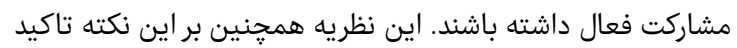

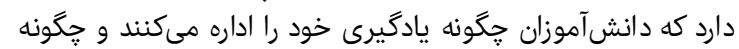

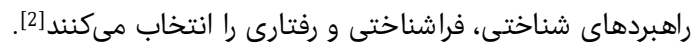

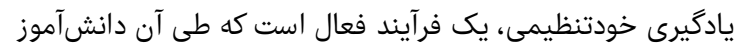

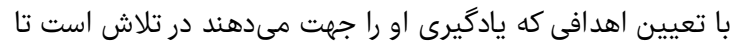

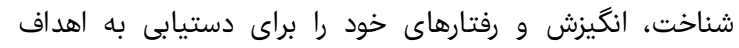

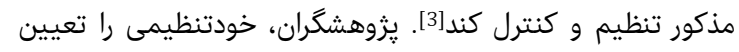

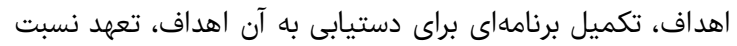

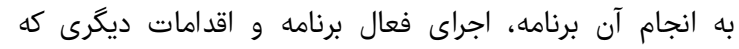

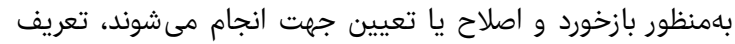

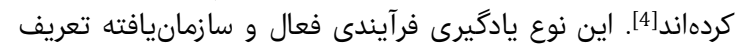

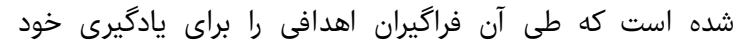

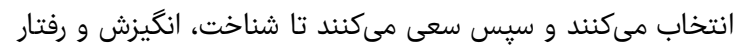

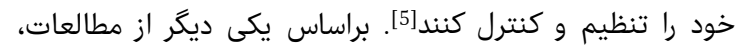

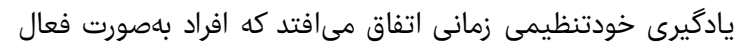

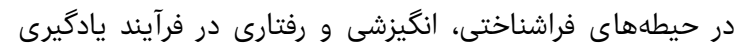

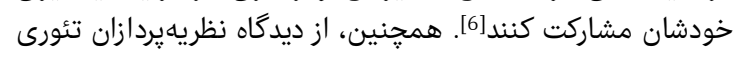

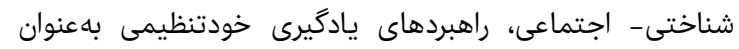

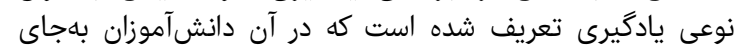

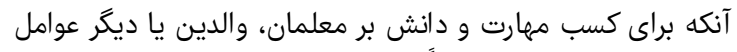

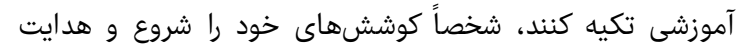

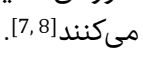

يافتهاهاى يزوهشى نشان دادهاند كه خودتنظيمى نقش مانش مهمى در دران

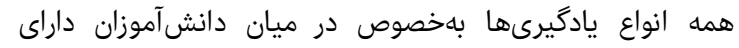

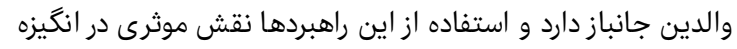

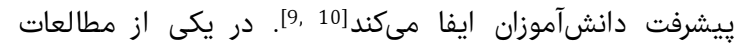

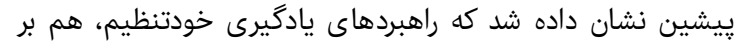

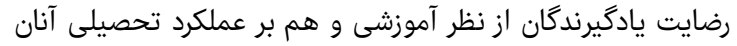

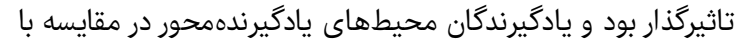

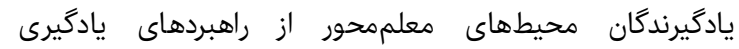

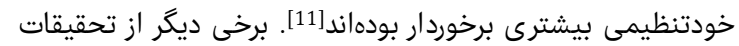

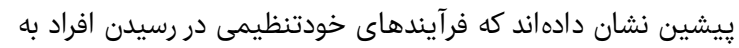

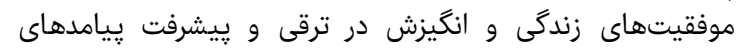

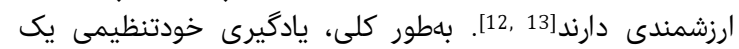

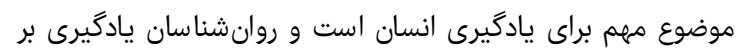

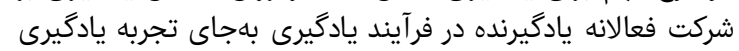

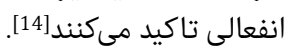

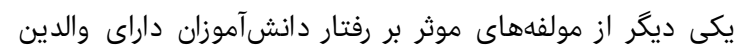

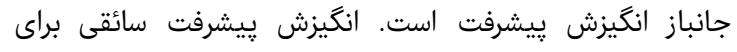

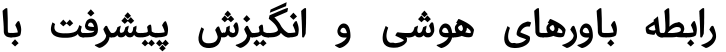

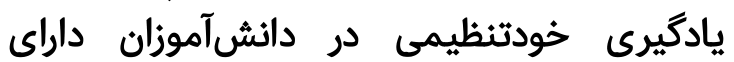

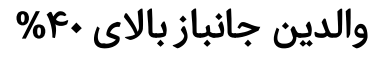

\author{
PhD " ابوطالب سعادتى شامير

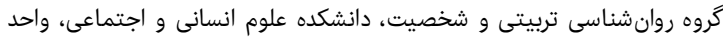

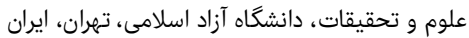

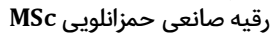

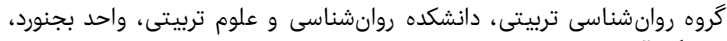 \\ دانشكاه آزاد اسلامى، بجنورد، ايران تران
}

حكيده

اهداف: دانشيده آموزانى كه فرزند جانباز هستند با مسايلى روبهرو مى شوند كه آنها

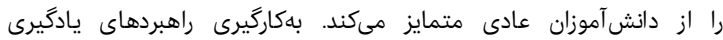

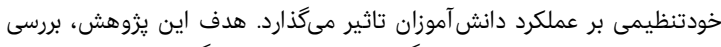

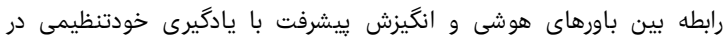

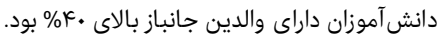

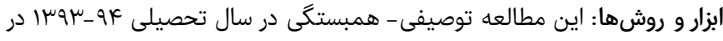

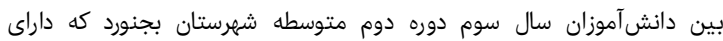

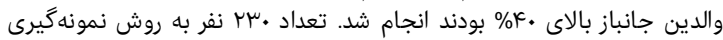

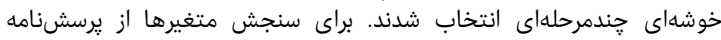

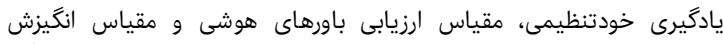

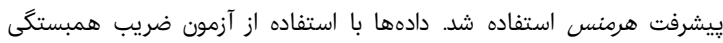

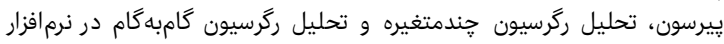
يافتها: SPS 23

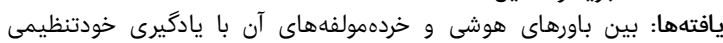

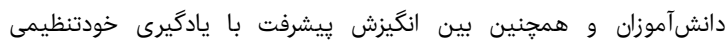

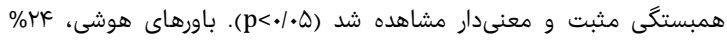

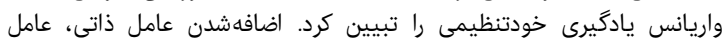

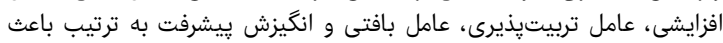

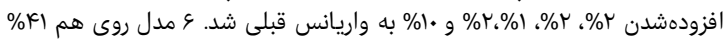

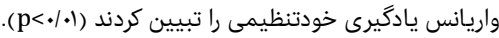
نتيجه

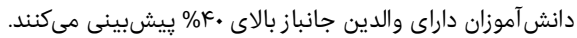

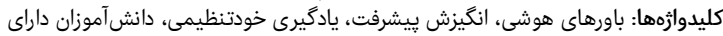

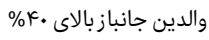

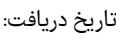

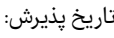

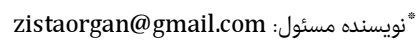

مقدمه - مق

مفهوم جانبازى يس از انقلاب اسلامى ايران و در دوران جنگ ايران

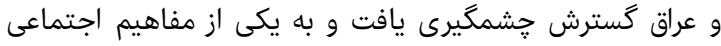

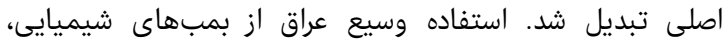

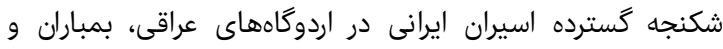

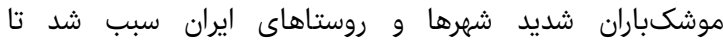

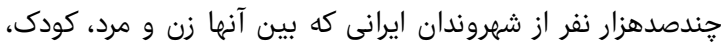

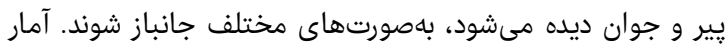

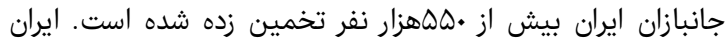

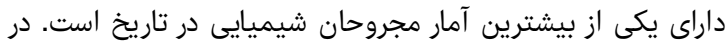

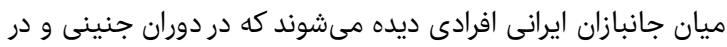

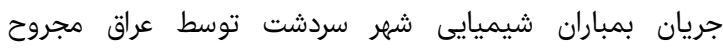

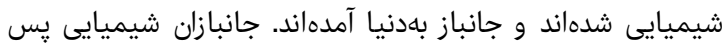

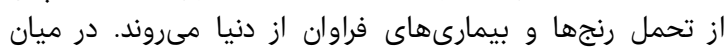
جانبازان ايرانى با معلوليتهاى مختلف، جانبازاز اندان اعصاب و روان دران 


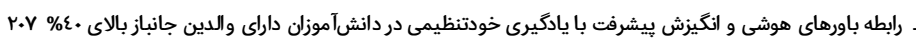

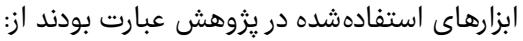

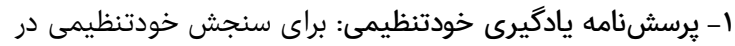

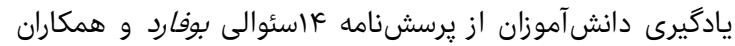

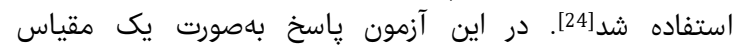

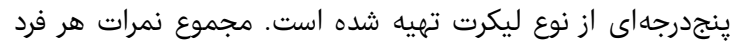

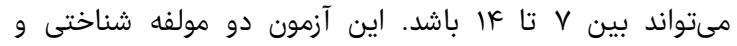

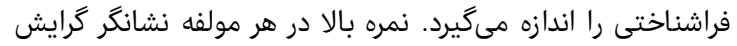

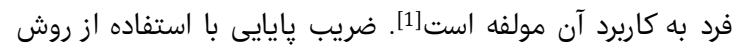

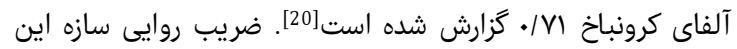

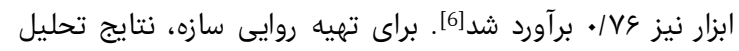

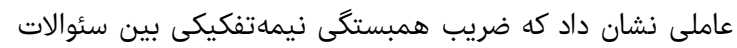

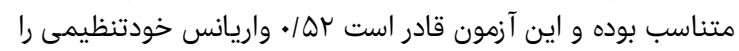

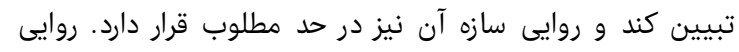

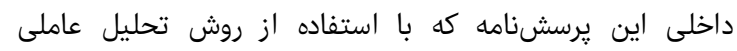

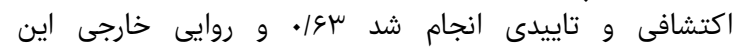

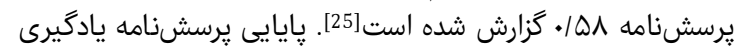

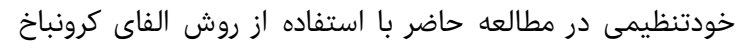

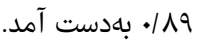

r- مقياس ارزيابى باورهاى هوشى: براى سنجش آد باورهاى هوشى

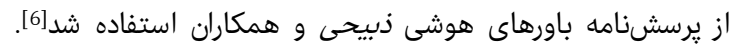

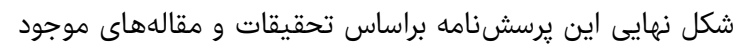

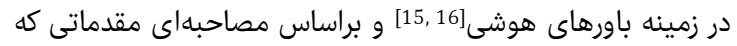

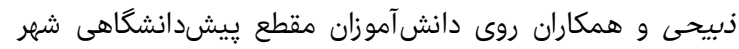

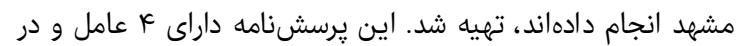

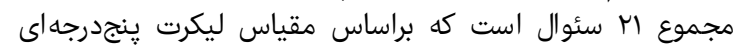

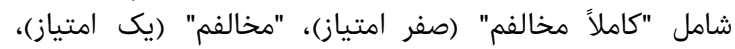

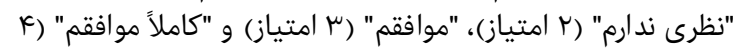

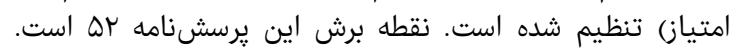

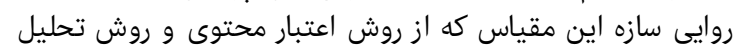

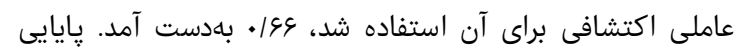

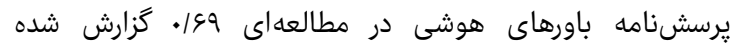

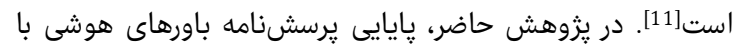

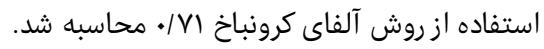

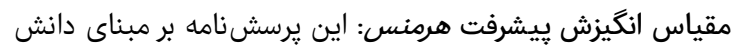

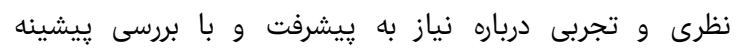

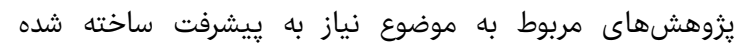

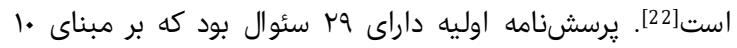

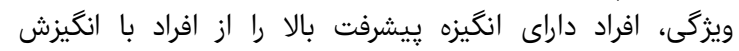

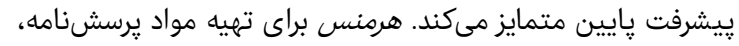

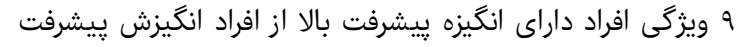

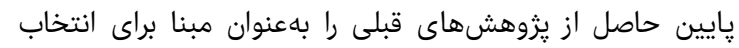

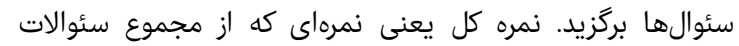

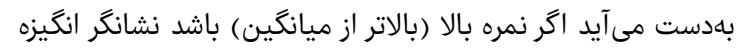

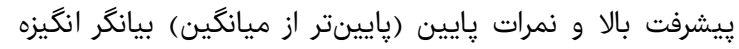

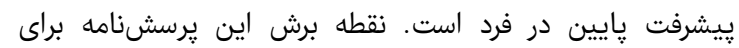

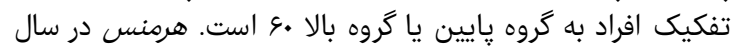

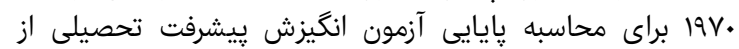

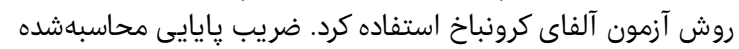

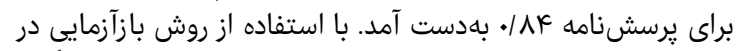

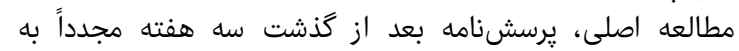

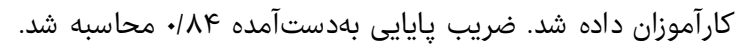

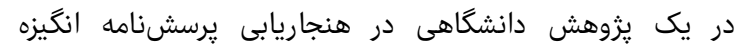

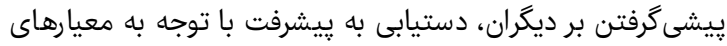

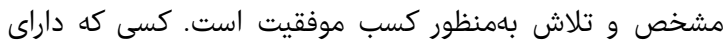

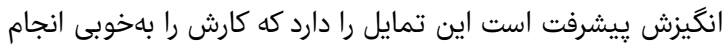

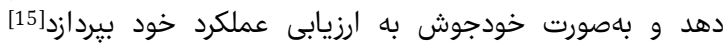

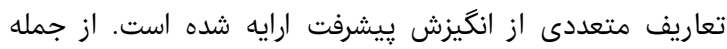

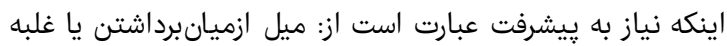

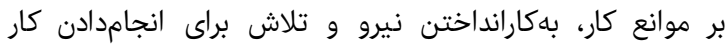

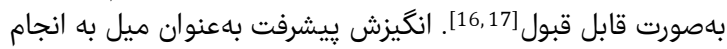

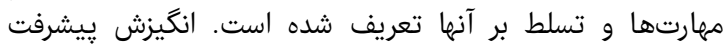

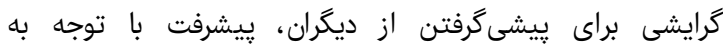

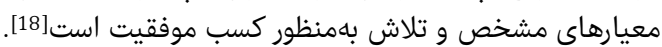

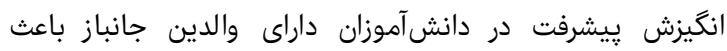

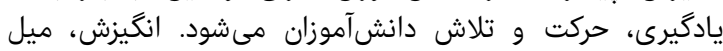

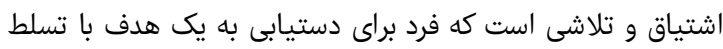

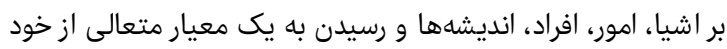

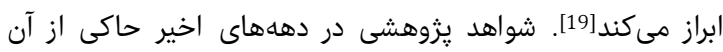

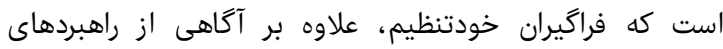

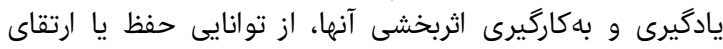

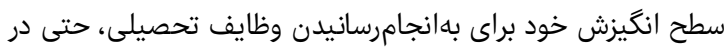

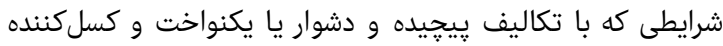

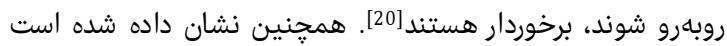

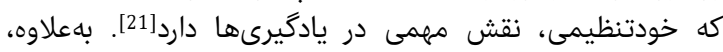

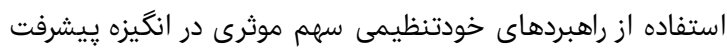

دارد[22].

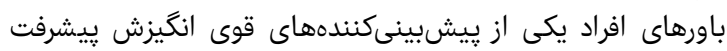

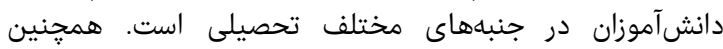

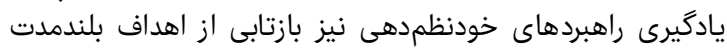

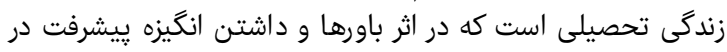

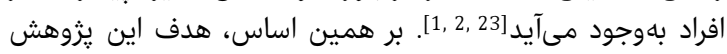

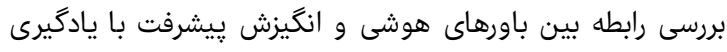

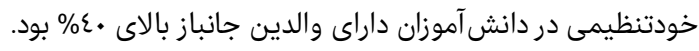

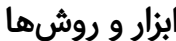

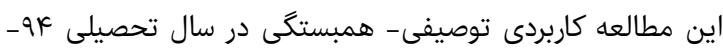

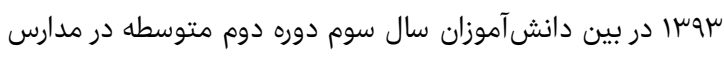

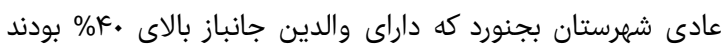

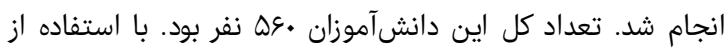

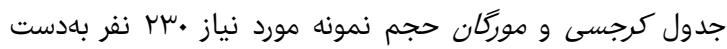

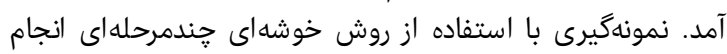

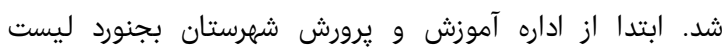

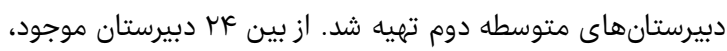

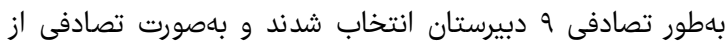

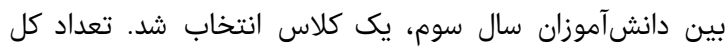

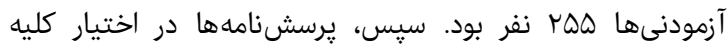

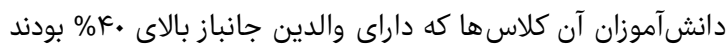

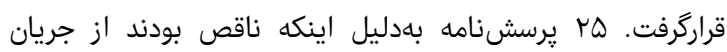

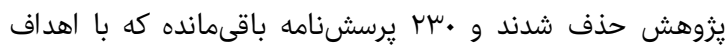

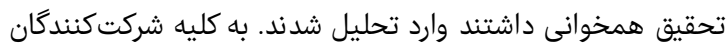

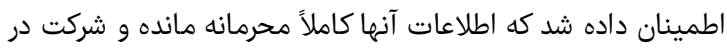
يثوهش هيج ضرر احتمالى براى آنها ندارد. 


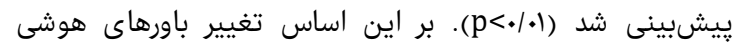

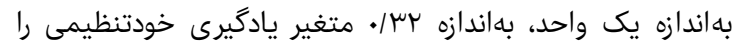

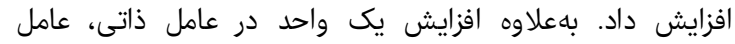

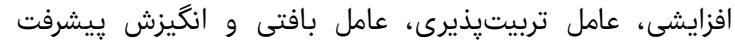

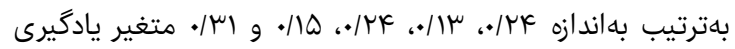
خودتنظيمى را افزايش دادند.

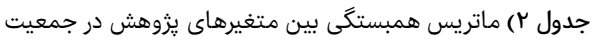

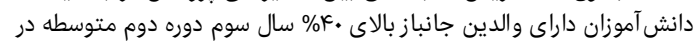

\begin{tabular}{|c|c|c|c|c|c|c|c|c|}
\hline$\wedge$ & 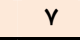 & 8 & $\Delta$ & $F$ & $\mu$ & $r$ & 1 & متغيرها \\
\hline & & & & & & & 1 & 1 - عامل ذاتى \\
\hline & & & & & & 1 & . $/ \pi \varepsilon^{*}$ & افزايشى \\
\hline & & & & & 1 & $\cdot / 1 \mathbb{N}^{* *}$ &.$/ r r^{* *}$ & "r-عامل \\
\hline & & & & 1 &.$|4|^{* *}$ &.$\mu *^{*}$ &.$/ 4 r^{* *}$ & F- عامل بافتى \\
\hline & & & 1 &.$/ r q^{*}$ &.$/ \& \Delta^{* *}$ &.$/ \Lambda^{* *}$ &.$/ \mu r^{* *}$ & هـ هاوشى باوراى \\
\hline & & 1 &.$|k|^{* *}$ &.$/ 1 *^{* *}$ & $\cdot \mid \Delta r^{* *}$ & $\cdot / 1 \mathbb{*}^{* *}$ & $\cdot / \mu \mu^{*}$ & 4-راهبردهاى \\
\hline & 1 & $\cdot \mu^{\mu *}$ & . $\mid \varepsilon \mu^{*}$ &.$/ I V^{*}$ & ק & $. \mu . *$ & $.119^{* *}$ & Vراشناختى V- راهبردهاى \\
\hline 1 & . $\mu^{* *}$ & $\cdot|r|^{*}$ & $\cdot / 1 \Lambda^{* *}$ & $\cdot|\omega|^{* *}$ & 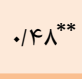 &.$/ 1{ }^{* *}$ &.$/ \mathrm{V}^{* *}$ & خودتنظيمى يادگيرى \\
\hline.$/ \Lambda^{*}$ & ${ }^{*} \cdot / / \mathrm{V}^{*}$ &.$/ r r^{*}$ &.$/ 1 K^{* *}$ & $\cdot \mid \Delta \mu^{* *}$ & . &.$/ 1 *^{* *}$ & $\cdot / r \Lambda^{* *}$ & 9يشرفتيزش \\
\hline
\end{tabular}

بحث

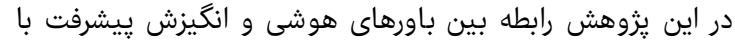

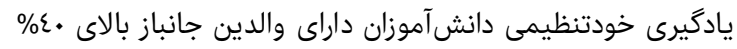

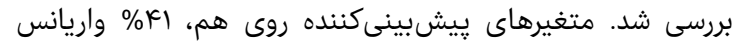

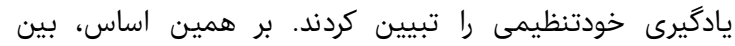

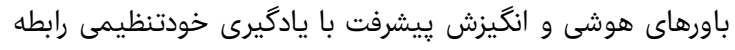

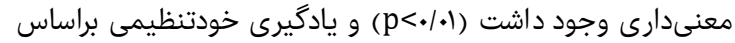

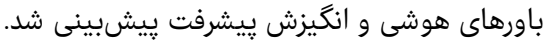

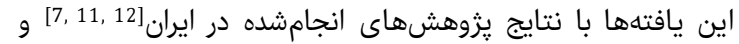

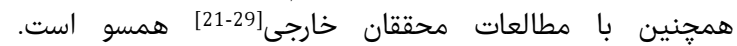

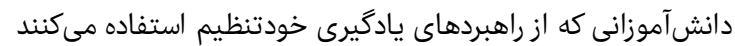

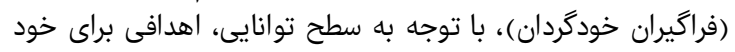

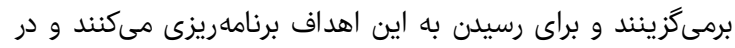

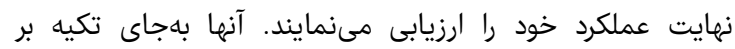

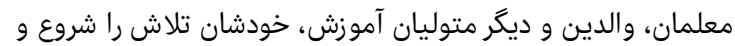

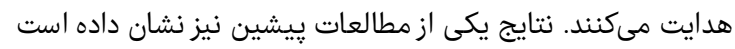

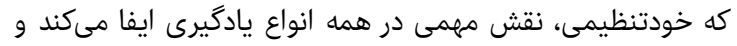

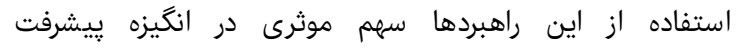

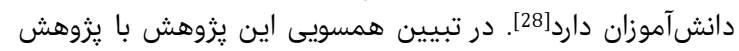

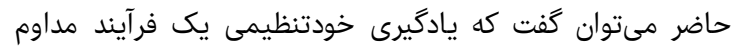

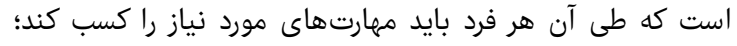

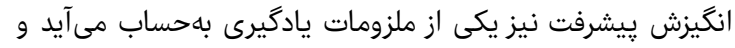

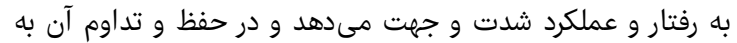

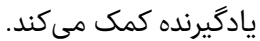

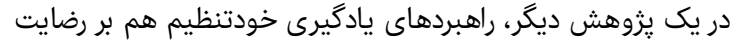
يادگيرندگان از نظر آموزشى و هم بر عملكرد تحصيلى رهئ آنان تاثيركذار

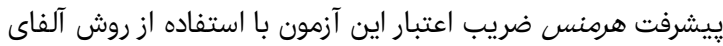

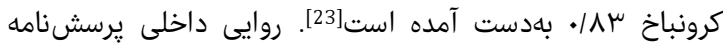

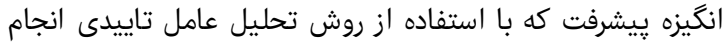

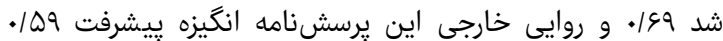

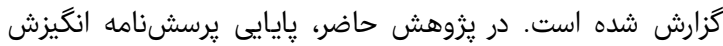

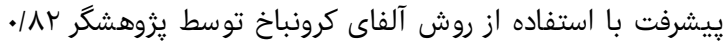

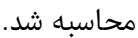

از نرمافزار SPSS 23 براى تجزيه و تحليل دادهها استفاده شد.

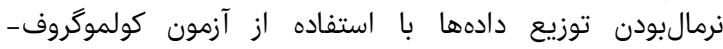

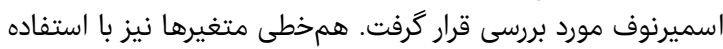

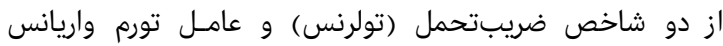

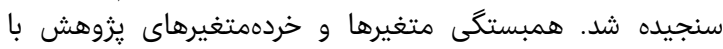

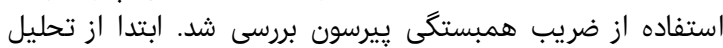

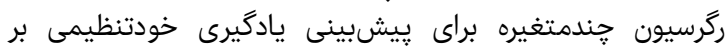

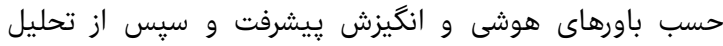

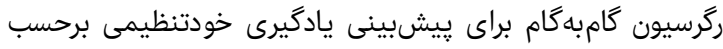

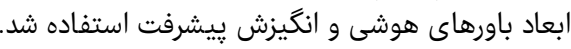

\section{يافتهها}

بالاترين و كمترين ميانكين نمره در ميان خردهمولفههاى باورهاى

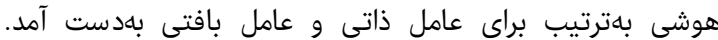

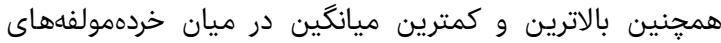

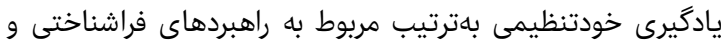
راهبردهاى شناختى بود (جدول () ).

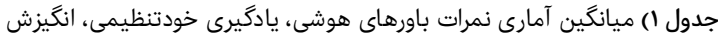

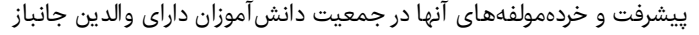

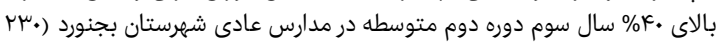
نفر در مان

\begin{tabular}{|c|c|c|c|}
\hline بيشترين & كمتريز & ن مرات & متغيرها \\
\hline & & & باورهاى هوشى \\
\hline$\mu k$ & 11 & $r F / r q \pm \mu / r F$ & عامل ذاتى \\
\hline IV & $\Lambda$ & $I V / Q \mu_{ \pm} I / T F$ & عامل افزايشى \\
\hline If & $\theta$ & $\mid V / \Delta \mu_{ \pm} \pm / / \mu$ & عامل تربيتيذيرى \\
\hline 1. & r & $\Lambda / \Delta K \pm \cdot / \Lambda K$ & عامل بافتى \\
\hline ve & KF & $\triangle V / T K \pm \Delta / Q V$ & 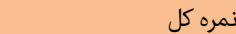 \\
\hline & & & يادكيرى خودتنظيمى \\
\hline$\mu_{1}$ & 10 & $r \Delta / \mu r \pm \mu / r F$ & راهبردهاى شناختى \\
\hline rr & IV & $r N / K V \pm \Psi / G K$ & راهبردهاى فراشناختى \\
\hline 94 & rF & $\Delta Q / \mu K_{ \pm} \varepsilon / / V$ & ن مره كل \\
\hline III & kI & $\Lambda \Lambda / \mu \sigma \pm g / \curlyvee \Lambda$ & انمرهيزش يِيشرفت \\
\hline
\end{tabular}

بين تمامى متغيرهاى يزوهش همبستگى مثبت و معنىدار

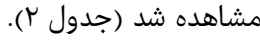

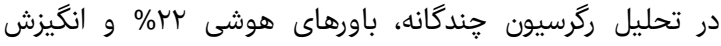

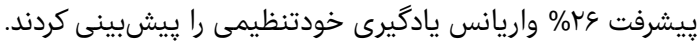

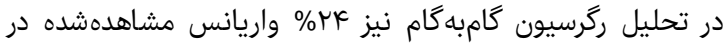

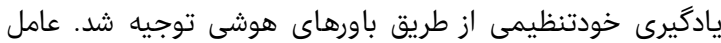

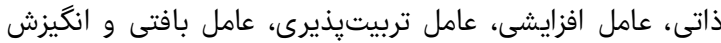

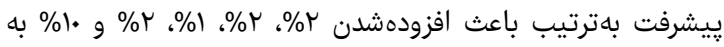

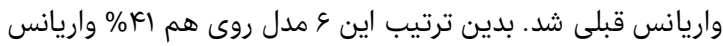

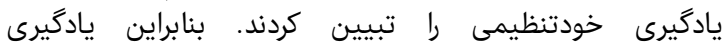

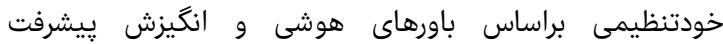




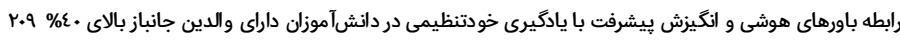

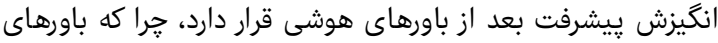

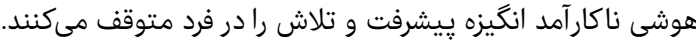

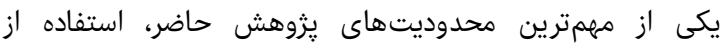

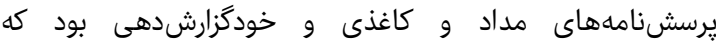

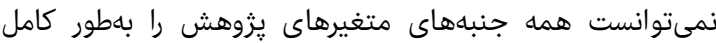

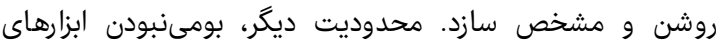

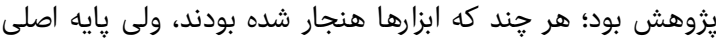

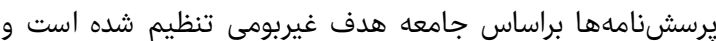

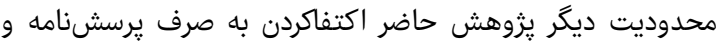

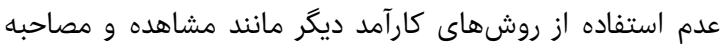

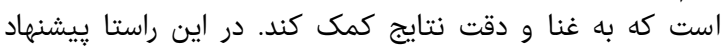

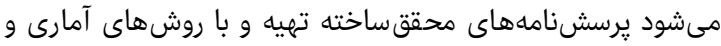

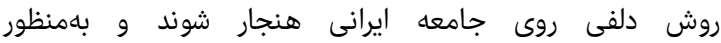

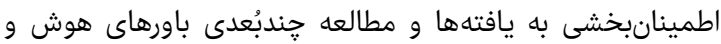

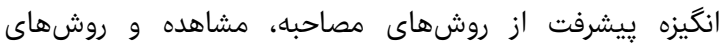

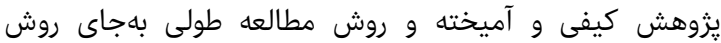
مقطعى نيز استفاده شود.

\section{نتيجه گليرى \\ باورهاى هوشى و انگيزش قيشيشرفت، يادگيرى خودتنظيمى را در

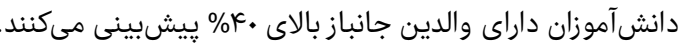

تشكر و قدردانى: از كليه دانشآموزان داراى والدين جانباز بالاى

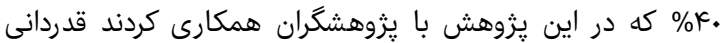

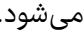

تاييديه اخلاقى: به كليه شركتكنندكان اطمينان داده شد كه

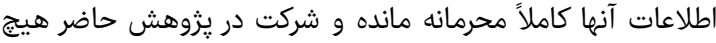
ضرر احتمالى براى آنها ندارد. تعارض منافع: موردى از طرف نويسندكان گزارش نشار نشده است.

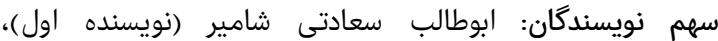

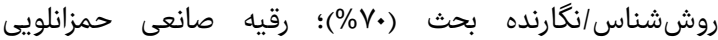

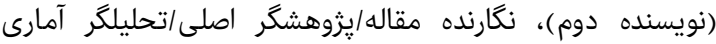

منابع مالى: منابع مالى اين يزوهش توسط نويسندكان تامين شد.

\section{منابع}

1- Mohzab A. Investigating the relationship between cognitive and metacognitive skills with Locus of control and achievement motivation in District 5 middle school students in Tehran [Dissertation]. Tehran: University of Tehran; 2014. [Persian]

2- Amani F, Amani A, Mohamadpour H. Comparison of motivational beliefs, self-regulation strategies and personality factors and their predictive role on the academic performance of gifted and normal students. J Women Family Stud. 2012;4(1):43-62. [Persian]

3- Hasani Zangbar T, Livarjani Sh. Investigating the effect of self- regulated learning strategies training on female first-grade high school students' math learning and anxiety in Bostan Abad in the academic year 2015-2016. J Instr Eval. 2017;10(3):69-93. [Persian]

4- Shokry O, Kadivar P, valiollah F, Sanghari AA, Zain Abadi $\mathrm{H}$, Ghanai $\mathrm{z}$, et al. The role of personality factors, learning approaches and thinking styles on academic

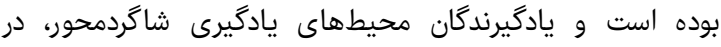

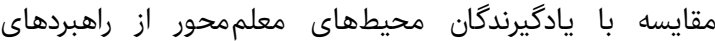

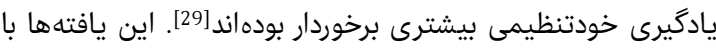

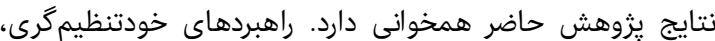

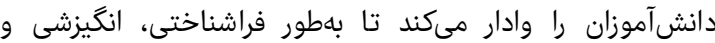

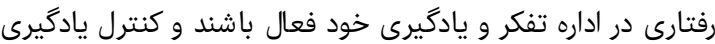

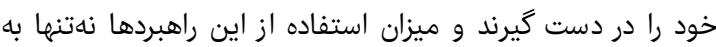

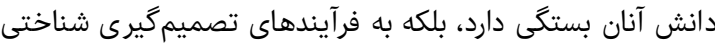

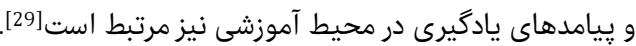

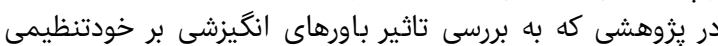

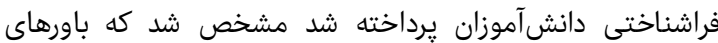

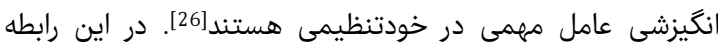

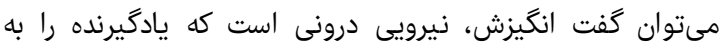

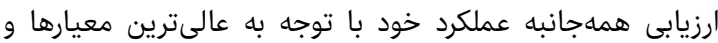

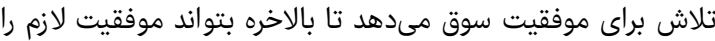

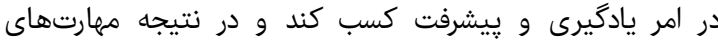
خودتنظيمى را بهبود بخشد.

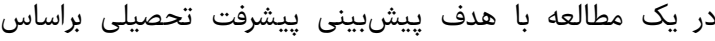

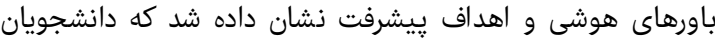
داراى باورهاى هوشى افزايشى، اهدافى تبحرى و دانشجويان داراى

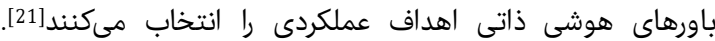

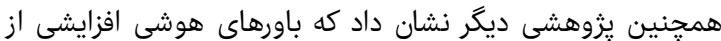
طريق واسطه گیى اهداف تبحرى، داراى اثرى غيرمستقيم و مثبت

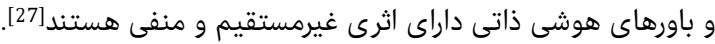

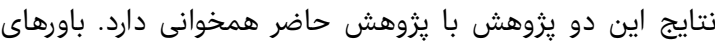

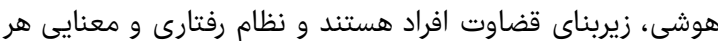

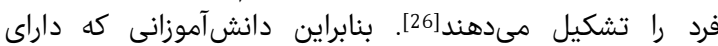

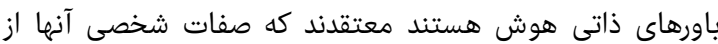

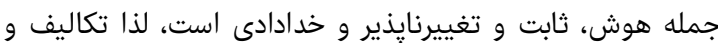

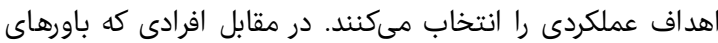

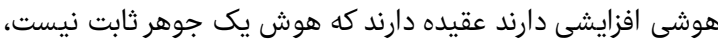

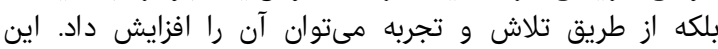

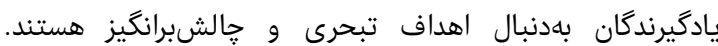

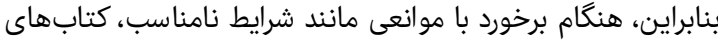

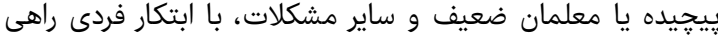

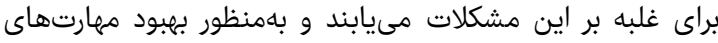
خودتنظيمى خود تلاش مىكنيند. بهطور كلى، علاقه به يادگيرى خودتنظيمى محصول عواملى است إنى

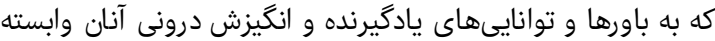

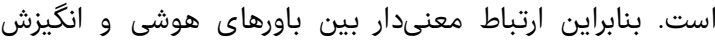

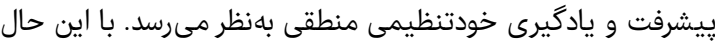
دانشآموزانى كه تصور مىكنند داراى هوش ذآني ذاتى بالايى هستند

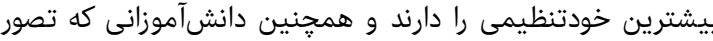

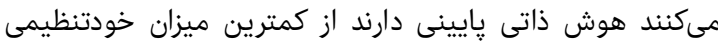

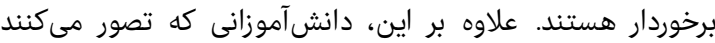

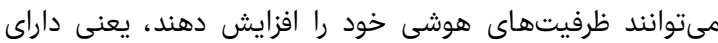

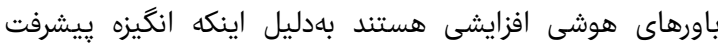

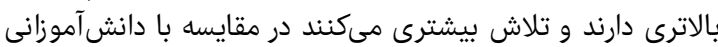

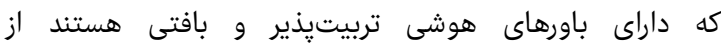

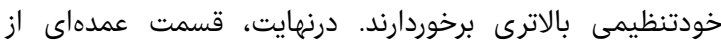

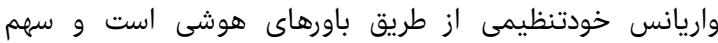


16- Abouserie R. Self-esteem and achievement motivation as determinants of students' approaches to studying. J Stud High Educ. 1995;20(1):19-26. [Persian] 17- Yasaminejad P, Taheri M, golmohammadian M, Ahadi $\mathrm{H}$. The relationship between self-regulation and achievement motivation and academic achievement of high school female students in Tehran. J Teach Learn.2013;20(3):325-38. [Persian]

18- Berger JL, Karabenick SA. Motivation and student's use of learning strategies: Evidence of unidirectional effects in mathematics classrooms. Learn Instruc. 2011;21(3):416-28.

19- Bidjerano T. Yun Dai D. The relationship between the big five model of personality and self-regulated learning strategies. Learn Individ Differences. 2007;17(1):69-81.

20- Boekaerts M, Zeidner M, Pintrich PR. Handbook of self- regulation. San Diego: Academic Press; 1999. pp. 416-28.

21- Bouchard B, Parent S, Lavirée S. Self- regulation on a concept information's task among average and gifted student. Excremental Child Psychol. 1993;56(1):115-34.

22- Schunk D H, Zimmerman B J. Self-regulated learning: From teaching to self-reflective practice. New York: The Guilford Press; 1998.

23- Deci E L, Ryan R M. The "what" and "why" of goal pursuits: Human needs and the self-determination of behavior. Psychol Inq. 2000;11(4):227-68.

24- Dembo M h. Applying educational psychology. $5^{\text {th }}$ edition. New York: Longman Publication Group; 1994.

25- Walton GM1, Spencer SJ. Latent ability: grades and test scores systematically underestimate the intellectual ability of negatively stereotyped students. Psychol Sci. 2009;20(9):1132-9.

26- Hadwin AF. Self-regulated learning. In: Good TL, editor. 21st Cntury eucation: A reference handbook. Thousand Oaks, CA: Sage Publications; 2008. pp. 175-83.

27- Singh K. Study of achievement motivation in relation to academic achievement of students. Inter J Educ Plann Prospective Valid. 2011;1(2).161-71.

28- Zhang LF. Further investigating thinking styles and psychosocial development in the Chinese higher education context. Learn Individ Differen. 2010;20(6):593-603.

29- Zimmerman BJ. Investigating self-regulation and motivation: Historical background, methodological developments, and future prospects. Am Educ Res J. 2010;45(1):166-83. achievement of students. J Psychol. 2007;11(4);447-70. [Persian]

5- Hajiyakhchali A, Morovvati Z, Fathi F. The relationship between personality characteristics, intelligence beliefs and academic achievement goals and self-efficacy. J Per Individ Difference.2014;3(4):75-92. [Persian]

6- Zabihi Hesari N, Ghulam Ali Lavassani M. The relationship between intelligence beliefs and goals of progress with self-efficacy in the students of the Payame Noor University. J Res Learn Virtual Sch. 2004;1(4):9-17. [Persian]

7- Samadi M. Survey students self-monitoring and parents learning: The study of gender and academic performance. J Psychol Educ. 2004;34(1):157-75. [Persian]

8- Yousefy A, Ghassemi Gh, Firouznia S. Motivation and academic achievement in medical students. J Educ Health Promot. 2012;1:423-33.

9- Mohamadameenee $\mathrm{Z}$. The relation between self regulated learning strategies, motivational beliefs, and students academic achievement. Q J New Thoughts Educ. 2008;4(4):123-36. [Persian]

10- Zahed A, Rajabi S, Omidi M. A comparison of social, emotional and educational adjustment and self-regulated learning in students with and without learning disabilities. J Learn Disabil. 2012;1(2):43-62. [Persian] 11- Samadi M. The effect of educational and selfregulatory methods on academic achievement. 2007;9(1):40-8. [Persian]

12- Vayskarami H, Sabziyan S, Pirjavid F, Gravand H. The relationship between perception of parenting styles and self-regulatory learning strategies of students at Ferdowsi University of Mashhad. J Res Virtual Learn. 2014;2(5):81-98. [Persian]

13- Jahromy Gh. The relationship between intelligence beliefs, achievement goals and cognitive engagement among high school students. J Educ Psychol. 2011;2(1):11-29. [Persian]

14- Kareshki, $\mathrm{H}$. The role of dimensions of perfectionism and goal orientations in self-regulation prediction of Shiraz University students. J Knowl Res Appli Psychol. 2013;14(2):100-8. [Persian]

15- Mahdian H, Ahadi H, Ahghari Gh. Standardize training persuasion questionnaire and its relationship with unbelievable intelligence and academic performance of middle scholar's city of Kashmar. J Educ Meas. 2013;1(2):23-36. 